Check for updates

Cite this: RSC Adv., 2017, 7, 23759

\title{
Ordered and disordered cyclodextrin nanosponges with diverse physicochemical properties $\uparrow$
}

\author{
Vikramjeet Singh, ${ }^{\text {ad }}$ Jianghui Xu, ${ }^{a}$ Li Wu, ${ }^{a}$ Botao Liu, ${ }^{a}$ Tao Guo, ${ }^{a}$ Zhen Guo, ${ }^{\text {ab }}$ \\ Peter York, ${ }^{\star a b}$ Ruxandra Gref ${ }^{\star c}$ and Jiwen Zhang (D) *a
}

\begin{abstract}
Herein, in addition to conventional $\beta$-CD-NSPs, the NSPs of $\alpha, \gamma$, HP- $\beta$, methyl $\beta$, and SBE- $\beta$ cyclodextrins were synthesized by a simple modified approach and thoroughly characterized. The control of CDs derivatization over structural dimensions and properties was clearly observed. It is interesting to note the complete transformation of $\beta-C D$ from its crystalline form to its non-crystalline derivatives and the further reversal when the derivatives are prepared into NSPs including those of $\alpha$ and $\gamma$-CDs (as observed by PXRD). The SEM images revealed the different morphologies and porous structure of NSPs and, in particular, the NSPs of methyl- $\beta-C D$ exhibited regular spherical shapes. Two drugs of different categories, doxorubicin and captopril, were evaluated for loading efficiencies, which were found to significantly vary with cross-linker ratio $(1: 4$ and $1: 6)$ and CD types. Together, all the synthesized NSPs provide a new horizon to try to solve existing problems relating to drug delivery.
\end{abstract}

Received 14th January 2017

Accepted 18th April 2017

DOI: $10.1039 / c 7 r a 00584 a$

rsc.li/rsc-advances

can be used to increase the dissolution rate and stability and

\section{Introduction}

Cyclodextrins (CDs) are a family of cyclic oligosaccharides with a hydrophilic outer surface and a lipophilic central cavity. ${ }^{1}$ The applications of CDs can be found in practically all sectors of industry; particularly, in the pharmaceutical industry, they are used as complexing agents to increase the aqueous solubility and bioavailability of poorly soluble drugs, compatibility, stability and taste-masking. Various CD derivatives, such as hydroxyl-propyl, sulfo-butyl-ether and methylated derivatives of $\beta$-cyclodextrins, have gained pharmaceutical interest due to their enhanced aqueous solubility and special physicochemical properties. $^{2}$ Recently, several new drug delivery systems including liposomes, microspheres, microcapsules, metal organic frameworks and nanosponges were designed based on cyclodextrins..$^{3-7}$

Cyclodextrin nanosponges (CD-NSPs), proposed as a new nanosized drug delivery system, are advanced cross-linked cyclodextrin polymers that are nanostructured within a threedimensional network. Because of their nano-porous structure, CD-NSPs can advantageously carry both water insoluble (Biopharmaceutical Classification System class-II drugs) and soluble drugs for safe and targeted delivery. ${ }^{\mathbf{8}, 9}$ These complexes

${ }^{a}$ Center for Drug Delivery Systems, Shanghai Institute of Materia Medica, Chinese Academy of Sciences, Shanghai 201203, China. E-mail: jwzhang@simm.ac.cn

${ }^{b}$ University of Bradford, Bradford, West Yorkshire BD7 1DP, UK

'Institute of Moléculaires Sciences d'Orsay, UMR CNRS 8214, Université Paris Sud, Université Paris-Saclay, 91400 Orsay, France

${ }^{d}$ School of Pharmaceutical Sciences, Sun Yat-Sen University, Guangzhou, China

$\dagger$ Electronic supplementary information (ESI) available. See DOI: $10.1039 / \mathrm{c} 7 \mathrm{ra} 00584 \mathrm{a}$ mask the unpleasant taste of drugs. Previously, researchers have mainly focused on the synthesis and the applications of $\beta$ CD-NSPs. Subsequently, the $\beta$-CD-NSPs were reported to be synthesized and used in a number of applications including loading of drugs, e.g., doxorubicin, resveratrol, camptothecin, paclitaxel, itraconazole, and in the drug delivery application. ${ }^{10-15}$ Apart from the pharmaceutics, NSPs have a wide range of applications including water purification, by removal of micropollutants, gas storage, and catalysis. ${ }^{16}$ Recently, a new type of NSPs through co-polymerization of $\beta$-CD with calixarene, benzylation and tetrafluoro-terephthalonitrile of $\beta$ CD was also synthesized, which showed high BET surface area and pollutant absorption ability compared to the conventional NSPs. ${ }^{17-19}$ Apart from $\beta$-CD, the other types of cyclodextrins or their derivatives possess different and special physicochemical properties including outer and inner cavity and solubility and hence, the ability to form inclusion complexes with different drugs. Very recently, the synthesis and characterization of polyamino cyclodextrin nanosponges showing $\mathrm{pH}^{-}$ dependent absorption abilities have been reported. ${ }^{20}$ Therefore, it is worth to try other commonly available CD derivatives for NSPs preparation and interesting to see how they alternate the physiochemical parameters of NSPs. There are a number of different techniques and cross-linkers reported for the synthesis of NSPs according to their applications. ${ }^{21}$ The intensive research on structural parameters of CD-NSPs including vibrational dynamics, sol-gel transitions, swelling phenomena and biodegradability is now well documented. ${ }^{22-25}$ However, it was hypothesized that the CD-NSPs of an amorphous or crystalline structure with regular and irregular 
shapes can be obtained, ${ }^{\mathbf{8}, 9}$ but not a single report was found in this regard. However, the perfectly cubic shaped $\gamma$-cyclodextrin NSPs was successfully synthesised in our lab using cyclodextrin metal organic frameworks as a template. ${ }^{26}$ Furthermore, the practical realisation of this hypothesis is much tougher due to the cross-linking nature of the reaction and large availability of hydroxyl groups in CDs. We envisaged that the derivatization of CDs could be used to control the structural parameters of the synthesized NSPs as the availability of hydroxide groups reduces after functionalization of CDs. In this direction, diphenyl carbonate was selected to synthesize the desired NSPs. NSPs of $\alpha, \beta, \gamma$, methyl- $\beta$, HP- $\beta$ and SBE$\beta$ CDs were synthesized by a simple and modified approach and thoroughly characterized using various techniques. For the proof of concept, two drugs with different physicochemical properties, doxorubicin $^{27}$ and captopril, ${ }^{28}$ were selected as model drugs to test and compare the loading efficiencies of the synthesized CD-NSPs. The detailed structural aspects for NSPs and their possible application and future direction are discussed in this report. It can be easily predicted that the new class of NSPs will open new possibilities to make efficient drug delivery systems.

\section{Experimental}

\section{Chemicals}

Unless otherwise noted, materials and solvents were obtained from commercial suppliers and used without further purification. Diphenyl carbonate (DPC) and trifluoroacetic acid were purchased from Aladdin Chemical. Dimethyl formamide (DMF), ethanol, methanol and TEA were ordered from Sinopharm Biotechnology Ltd. Acetonitrile of HPLC grade was obtained from Merck Co., Ltd. (Darmstadt, Germany). Water was purified by the Milli-Q system (Millipore, Bedford, MA, USA). Doxorubicin and captopril were purchased from Dalian Meilun Biotech Co., Ltd. (China). Hydroxypropyl- $\beta$-cyclodextrin (HP- $\beta$ CD, degree of substitution - 4.5) was obtained from Shijiazhuang Pharmaceutical Group Co., Ltd (Shijiazhuang, Hebei, China). Methyl- $\beta-C D(M-\beta-C D$, degree of substitution - 5 ) and sulphobutyl ether- $\beta$-CD (SBE, degree of substitution - 5) were purchased from Wacker Chemie AG and Cydex Pharmaceuticals Inc., respectively.

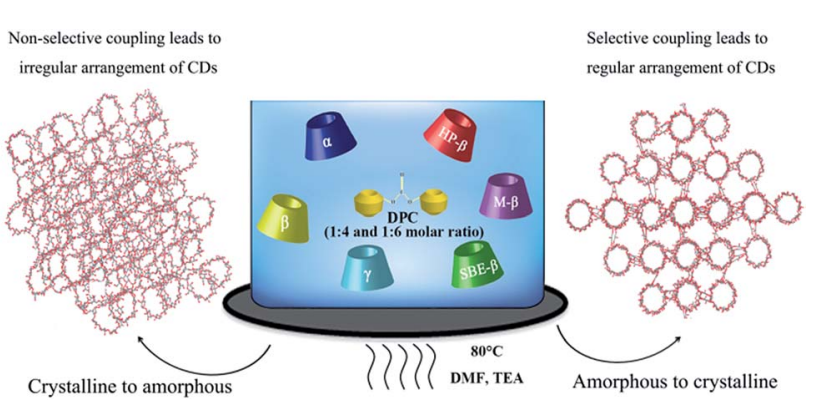

Fig. 1 Schematic of the reversal of ordered and disordered molecular arrangements in CD-NSPs synthesis.

\section{Synthesis of NSPs}

The schematic for the synthesis and effect on molecular arrangements is represented in Fig. 1. For the synthesis process, $60 \mathrm{mM}$ solutions $(10 \mathrm{~mL})$ of each type of $\mathrm{CD}$ (anhydrated), i.e. $\alpha$ (0.583 g), $\beta$ (0.681 g), $\gamma(0.778 \mathrm{~g})$, methyl- $\beta$ (0.786 g), HP$\beta(0.825 \mathrm{~g})$ and SBE- $\beta$ CDs (0.855 g) in DMF (anhydrated) were prepared in clean, dry round-bottomed flasks and heated to $80{ }^{\circ} \mathrm{C}$ to obtain clear solutions (turbid in case of $\alpha$ and SBE- $\beta$ ). Furthermore, $240 \mathrm{mM}(0.514 \mathrm{~g}, 1$ : 4) and $360 \mathrm{mM}(0.771 \mathrm{~g}, 1$ : 6) of diphenyl carbonate was added subsequently to each flask and stirred for $10 \mathrm{~min}$. Finally, $300 \mu \mathrm{L}$ of triethylamine $\left(\mathrm{Et}_{3} \mathrm{~N}\right.$, $\mathrm{C}_{6} \mathrm{H}_{15} \mathrm{~N}$ ) was also added to each flask, followed by stirring at 400 RPM for $3 \mathrm{~h}$. Triethylamine was used as a catalyst to facilitate the reaction and help to shorten the lengthy reaction time to just $3 \mathrm{~h}$. Specifically, $\mathrm{Et}_{3} \mathrm{~N}$ acts as nucleophile and opens up the carbonate ester of DPC and facilitates the proton exchange, which finally results in fast esterification of CD hydroxyl groups. After completion of the condensation polymerization reaction, gelation was observed. The reaction mass cooled to room temperature and 6 volumes of distilled water were added to get the precipitate of the respective NSP. All NSPs were then thoroughly washed by slurry in ethanol (Soxhlet extraction, $8 \mathrm{~h}$ ) and distilled water to remove unreacted reagents and byproducts, particularly phenol. The NSPs were subsequently lyophilized for $12 \mathrm{~h}$ using a freeze-dryer (SIM International Group Co., Ltd., USA). The amount of catalyst (TEA) and reaction time ( $3 \mathrm{~h}$ ) were set at minimal values required for NSPs production after complete validation.

\section{Characterization of NSPs}

FTIR spectroscopy of NSPs was recorded using ThermoScientific System Nicolet ${ }^{\mathrm{TM}}$ iS ${ }^{\mathrm{TM}} 5$ FT-IR spectrophotometer in the range from 4000 to $650 \mathrm{~cm}^{-1}$. The IR spectra of diphenyl carbonate (graph not shown) and each type of CD were also recorded as controls to compare with those from the nanosponges for structure confirmation. ${ }^{13} \mathrm{C}$ NMR spectra were recorded on an Agilent DD2 600 solid state NMR instrument equipped with a $15 \mathrm{kHz}$ rotating MAS probe.

After the washing process, all NSP samples were evaluated for size distribution, polydispersity index (PDI) and zeta potential in water using a Malvern Zetasizer. The samples, after freeze drying, were used to determine the real morphology of the nanosponges using the scanning electron microscopy (SEM) technique. The morphologies of various NSPs were examined with high-resolution field-emission SEM (3400N, Hitachi). Each type of NSP was sputtered with gold at a current of $15 \mathrm{~mA}$ for 3 min using an ion sputter. The freeze dried samples were used for powder X-ray diffraction (PXRD) analysis to retain the original internal structure. The particles were examined by PXRD using a D8 Advance (Bruker, Germany) with a locked coupled scan type. The scan speed was $0.1 \mathrm{~s}$ per step and the increment was $0.021^{\circ}$. The running conditions were $40 \mathrm{kV}, 40 \mathrm{~mA}$, and the scan range was $3-40^{\circ}$. Nitrogen adsorption-desorption isotherm was measured with a liquid nitrogen bath $\left(-196^{\circ} \mathrm{C}\right)$ using a porosimeter (TriStar 3000 V6.05 A, USA). In order to remove the interstitial solvents, the samples were activated by 
immersing in DCM for three days and drying under vacuum at $50{ }^{\circ} \mathrm{C}$ for $12 \mathrm{~h}$. Known amounts of samples (e.g. 150-200 mg) were loaded into the BET sample tubes and degassed under vacuum (10-5 Torr) at $100{ }^{\circ} \mathrm{C}$ for $5 \mathrm{~h}$. The BET model was applied to measure the specific surface area of the samples.

The thermo-gravimetric analysis (TGA) profiles were recorded using a differential scanning calorimeter (DSC 822, Mettler Toledo, Switzerland). About $2.0 \mathrm{mg}$ of each sample was heated in a pierced aluminium pan from $50{ }^{\circ} \mathrm{C}$ to $300{ }^{\circ} \mathrm{C}$ at a heating rate of $10{ }^{\circ} \mathrm{C} \mathrm{min}^{-1}$. Thermal data analysis of the DSC profiles was conducted using the Mettler Toledo STAR system software. TGA was carried out in the Netzsch TG 209 F3 equipment, using dry air with a nitrogen gas flow of $20 \mathrm{~mL} \mathrm{~min}^{-1}$ and a scan rate of $10{ }^{\circ} \mathrm{C} \mathrm{min}^{-1}$.

\section{Drug loading}

Two drugs with different physicochemical properties, doxorubicin and captopril, were taken into account for the optimization of drug loading efficiencies for synthesized NSPs. Distilled water was used as a medium for loading experiments for doxorubicin and captopril due to good aqueous solubility, which is safest among all. In loading experiments, $100 \mathrm{mg}$ of all types of NSPs were incubated with a water solution $(2 \mathrm{~mL})$ of $0.5 \mathrm{mg} \mathrm{mL} \mathrm{m}^{-1}$ (doxorubicin) and $5 \mathrm{mg} \mathrm{mL}^{-1}$ (captopril) drugs. After shaking for $24 \mathrm{~h}\left(100 \mathrm{rpm} \mathrm{min}{ }^{-1}\right)$ at room temperature, the samples were withdrawn and centrifuged (4800 rpm), and the supernatant was evaluated for loading capacity by HPLC. The concentration of drugs and shaking were also chosen after validation. The HPLC methods were different for specific drugs and are listed in the ESI. $\dagger$

\section{Results and discussion}

\section{Chemical characterization}

The FTIR spectra of NSPs were recorded (Fig. 2) with the relative CDs for chemical structure confirmation. It is easy to interpret the successful cross-linking of CDs from the FTIR spectra. The FTIR spectra from both linker ratios (1:4 and 1:6) were observed to be very similar. The appearance of the new peak of the carbonyl $(\mathrm{C}=\mathrm{O})$ group at $1754 \mathrm{~cm}^{-1}$ in NSPs spectra confirmed the successful cross-linking of relative CDs by DPC in various ratios. A broad peak at $1260 \mathrm{~cm}^{-1}$ (marked by a black arrow in Fig. 2) represents the $\mathrm{CD}-\mathrm{O}-\mathrm{C}-\mathrm{O}$ stretching in the nanosponge structure. In addition to the FTIR, ${ }^{13} \mathrm{C}$ NMR test
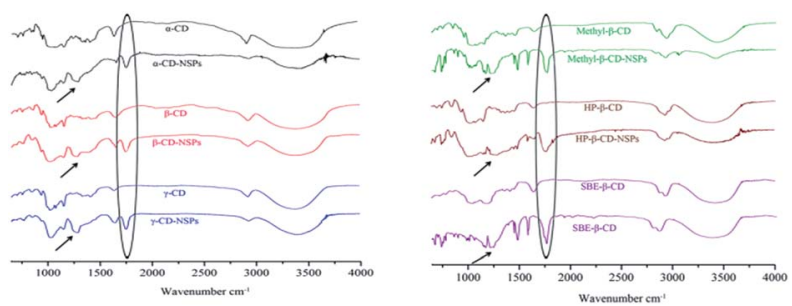

Fig. 2 FTIR spectra of $\alpha, \beta, \gamma$-CD and $\beta$-CD derivative NSPs (left) in comparison with their monomers (right) for chemical structural analysis. The specific peaks for carbonyl in the NSP spectra are marked. was also performed on $\gamma$-CD-NSPs types for further structural confirmation and to ensure the NSPs' purity. The obtained spectra, presented in Fig. S1, $\dagger$ showed two new peaks at 31.9 and $32.4 \mathrm{ppm}$ compared to that of $\gamma$-CD, thus confirming the successful linkage of primary as well as secondary hydroxyl groups. The fact that no other impurities were detected in the spectra showed that the sponges were purified and all related byproducts were removed successfully.

\section{Size distribution and zeta potential of NSPs}

A detailed report of particle size, PDI and zeta potential is presented in Table S1. $\uparrow$ The dynamic light scattering (DLS) related measurements were carried out before lyophilization and after the washing process. Overall, the size of NSPs with a CD-linker ratio of $1: 4$ was found to be extremely low compared with the size of NSPs with a CD-linker ratio $1: 6$, possibly due to the cross-linking efficiency of the system during the polymerization reaction. The size of $\alpha$-NSPs $(1: 4)$ and $\gamma$-NSPs $(1: 6)$ were recorded to be smallest $(327.6 \mathrm{~nm})$ and largest $(2606 \mathrm{~nm})$, respectively. The overall sizes of NSPs found in the submicron range might be due to charge accelerated aggregation and molecular nature of relative CDs (except M- $\beta$-CD-NSPs), resulting in a size increment. The aggregation was found to increase during the drying process, which was later confirmed by SEM characterization also. Generally, in water or other polar solvents, the adsorption of anionic surfactants induce negative zeta potential. However, the charge on the particle is still dependent upon the structural composition of the particles themselves to some extent, which is reflected in the zeta potential measurements of NSPs (positive values) presented in Table S1.†

\section{Morphologies of NSPs}

Thus far, there have been very few studies reporting the detailed morphology of all the types of CD nanosponges, and those that do exist are limited to the $\beta$-CD NSPs only, as mentioned above. A complete structural characterization was carried out to reveal the real structure of CD-NSPs in this research. Since CD-NSPs are very delicate sponge-like structures, we envisaged that the morphology of CD-NSPs would change after the drying process

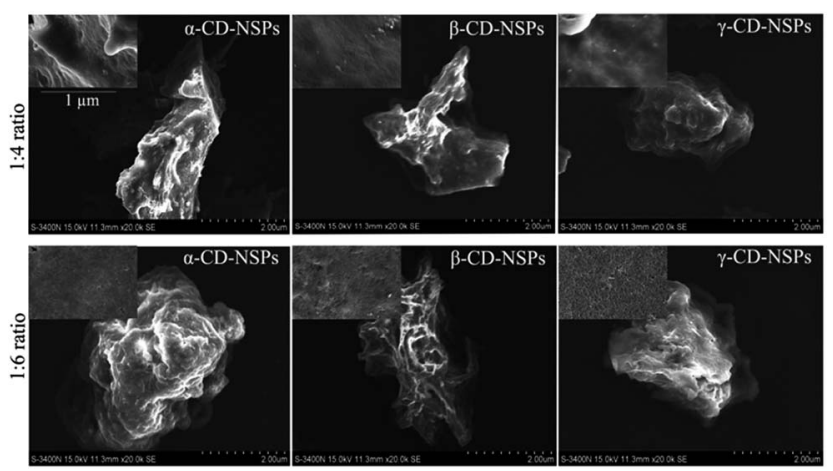

Fig. 3 SEM images of parent CD-NSPs with $1: 4$ and $1: 6$ cross-linker ratio. Insets show the surface view at a smaller scale. 


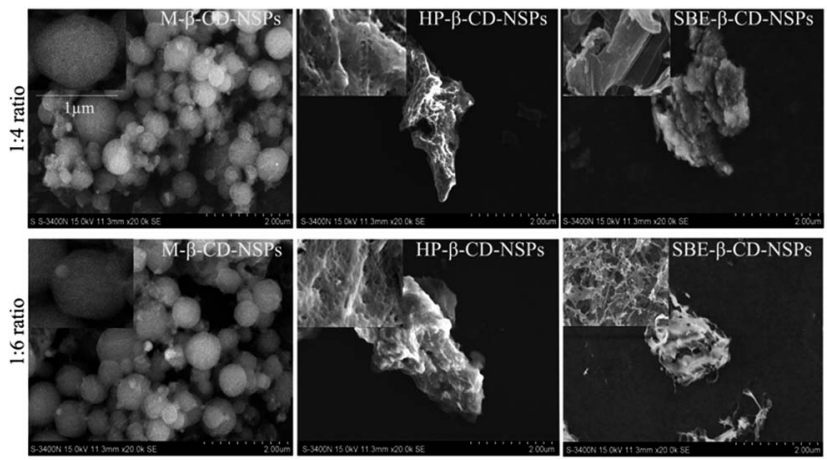

Fig. 4 SEM images of $\beta$-CDs derivative NSPs with $1: 4$ and $1: 6$ crosslinker ratio. Insets show the surface view at a smaller scale.

and the real morphology would be lost. Therefore, SEM images of oven-dried and lyophilized samples were recorded. As expected, a clear and significant difference was found in images of oven-dried and lyophilized CD-NSPs samples. The structures of oven-dried NSPs (single ratio, $1: 4$ ) are shown in Fig. S2. $\dagger$ As we can clearly see in Fig. 3 and 4, the lyophilized CD-NSPs were able to keep the real morphology, unlike the oven-dried CD-NSPs which appeared to shrink and form a hard cluster (Fig. S2 $\dagger$ ). It could be easily inferred that the real morphologies of CDNSPs were completely lost after drying in the oven, and no differences were found between them. The porous surfaces of NSPs can be clearly observed in SEM images of the lyophilized samples.

As shown in Fig. 3 and 4, the morphology of each type of lyophilized CD-NSPs was very different and of irregular shape, except M- $\beta$-CD-NSPs which exhibited a very regular, round and ball-like structures due to the hydrophobic nature of the parent CDs, reported for the first time herein. Moreover, the surfaces of CD-NSPs were closely monitored and a highly porous nature was recorded with a powerful SEM instrument. The morphology of a drug carrier greatly influences the drug loading capacity and bioavailability of the loaded drug. Thus, the new class of CD-NSPs will surely improve drug delivery efficiency.

\section{PXRD evaluation of NSPs}

Diffraction peaks in PXRD are useful for the determination of molecular arrangements within the given structure. It is of great interest to know the crystal nature of a drug carrier as it influences the drug loading capacity and release behaviour. Previously, Ansari et al., confirmed by PXRD that after lyophilization process, the crystallinity of CD-NSPs was lost, and it resulted in a 'fluffy' structure. Perhaps, the amorphous structure of $\beta$-CDNSPs in previous reports ${ }^{\mathbf{1 3}}$ was able to give some crystalline pattern after being compressed. In fact, the regular arrangements of CDs monomers was absent and even not possible due to cross-linking nature of the reaction.

However, our results showed that the above statement cannot be applied in all cases and varies with the NSP type. The PXRD spectra of CDs and their NSPs (after lyophilization) are presented in Fig. 5. The PXRD spectra of CDs showed the crystalline nature of the parent CDs and amorphous behaviour

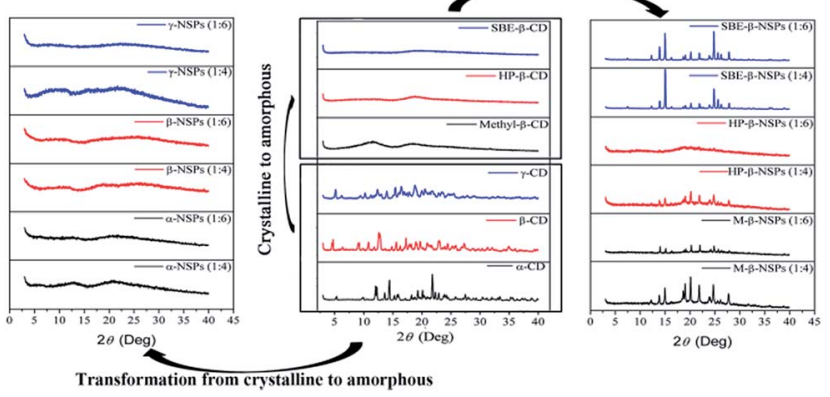

Fig. 5 Comparison of the PXRD patterns of all CDs, parent CDs-NSPs and their derivative NSPs showing transformation of crystallinity.

of derivative CDs. However, the PXRDs results of NSPs were found to be reversed and all parent CDs existed in highly amorphous state and possibly carried random arrangements of CDs. Interestingly, all the $\beta$-CD derivatives, including HP- $\beta$ NSPs, methyl- $\beta$-NSPs and SBE- $\beta$-NSPs, were transformed into crystalline structures, except HP- $\beta$-NSPs at a cross-linker ratio of $1: 6$ with less crystallinity, which might be due to the participation of hydroxyl-propyl groups in the cross-linking reaction at the higher linker (DPC) ratio. Possibly, the substitution degree has played an important role and a limited number of hydroxyl groups are available in the derivatives, which give rise to the regular arrangements in the respective NSPs. On the other hand, all of the hydroxyl groups are available for cross-linking in each parent CD resulting in irregular arrangements. The crystalline arrangements of $\beta$-CD derivatives depend upon the degree of cross-linking. It was concluded that the crystallinities of CD-NSPs were not totally dependent upon the drying process but on the degree of cross-linking, which controls the molecular arrangement.

\section{Thermal behaviour and nitrogen absorption evaluation}

The DSC and TGA curves of CD-NSPs $(1: 4)$ are shown in Fig. 6. The DSC and TGA patterns of NSPs with $1: 4$ ratio are presented only because no significant differences were recorded between $1: 4$ and $1: 6$ cross-linker ratios. The thermal transitions of CDNSPs can be explained from the DSC graph shown in Fig. 6A. It is very important to note that the DSC patterns of M-NSPs and SBE-NSPs are quite different from the other NSPs types. The
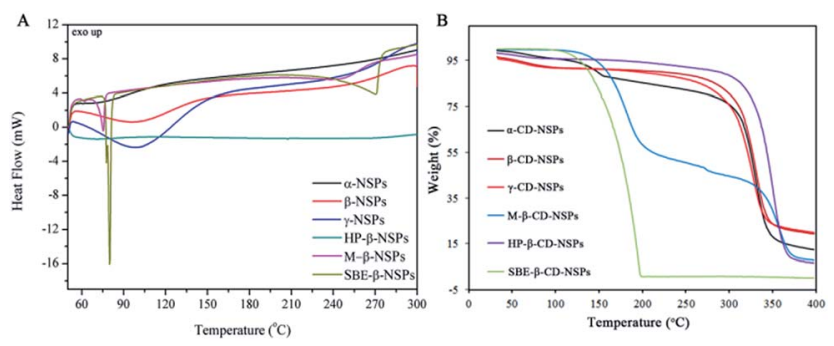

Fig. 6 (A) DSC graphs of all CD-NSPs (1:4) with zooming of the dip at $207^{\circ} \mathrm{C}$ to explain the crystalline nature of HP- $\beta-C D-N S P S$ and (B) TGA graph of all the CD-NSPs (1:4). 
strong peaks, which are usually considered as the crystallization temperature of polymers (Tc), were recorded at $72{ }^{\circ} \mathrm{C}$ and $79{ }^{\circ} \mathrm{C}$ for M- $\beta$-CD-NSPs and SBE- $\beta$-CD-NSPs, respectively. The crystalline nature of M- $\beta$-CD-NSPs and SBE- $\beta$-CD-NSPs was already confirmed from PXRD. However, no such peak was reported in case of HP- $\beta$-CD-NSPs and the TGA graphs shown in Fig. 6B mainly consist of a two-step process. Generally, the first step can be understood to occur due to the loss of solvent and the second may account for most of the weight loss and is associated with the formation of the residue of the CDs polymer. The second step shows the degradation of NSPs, which took place at a higher temperature between $300-350{ }^{\circ} \mathrm{C}$ for the parent CDs NSPs. The degradation behaviour of derivative CD-NSPs is recorded to be quite different from the parent CDs and even from each other, which might be due to change in the physicochemical properties after derivatization. The results for NSPs of HP- $\beta$-CD were quite different from those of the parent CDNSPs. The SBE- $\beta$-CD-NSPs were found to be unstable and degraded at $200{ }^{\circ} \mathrm{C}$, whereas the NSPs of methyl- $\beta$-CD were found to be the least stable and showed strange degradation in two steps at $185{ }^{\circ} \mathrm{C}(54 \%)$ and $360{ }^{\circ} \mathrm{C}(39 \%)$. The degradation and weight loss percentage of the CD-NSPs are clearly mentioned and shown in the DTG curves in Fig. S3. $\dagger$

All samples of NSPs were subjected to the BET measurement for specific surface area determination via the nitrogen absorption method. As expected, all NSPs showed poor nitrogen absorption and hence low specific surface area in the range of $0.325 \mathrm{~m}^{2} \mathrm{~g}^{-1}$ to $4.895 \mathrm{~m}^{2} \mathrm{~g}^{-1}$ with pore sizes of $2-40 \mathrm{~nm}$ width. The obtained results were in agreement with those reported in the literature for other cyclodextrin-based co-polymer materials. ${ }^{16}$

\section{Drug loading comparison}

To prove the hypothesis that the cross-linking ratio and the type of CDs could largely improve the loading capacity of NSPs, doxorubicin and captopril were selected as model drugs for payload comparison. Doxorubicin and captopril are anticancer agents and angiotensin-converting enzyme (ACE) inhibitors with molecular weights of 543.5 and 217.2, respectively (see the molecular structure in Fig. S4 $\dagger$ ). Herein, the payload efficiencies of all NSPs for drug delivery purposes were thoroughly measured and are compared. The loading efficiency of all NSPs for each drug is presented in Fig. 7. As expected, in addition to the different cross-linker ratio, the monomers (specific CDs) altered the loading efficiency to a large extent. Despite the exact

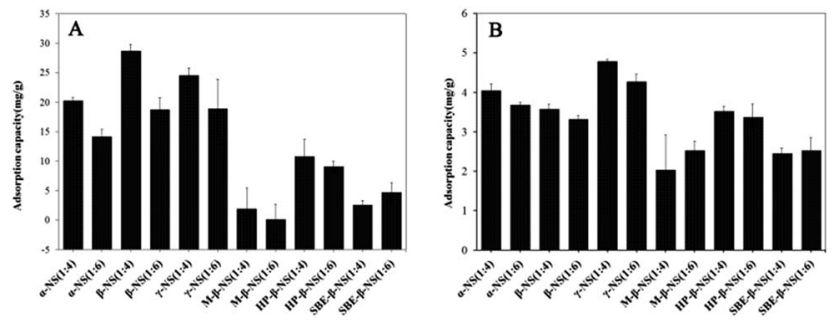

Fig. 7 Loading efficiencies of relative NSPs for (A) doxorubicin and (B) captopril. reason for the specific drug-NSPs combination for payload purpose being difficult to discuss due to the large number of combinations, the major points are addressed herein. A general tendency can be easily traced in the drug loading data indicating that the NSPs with $1: 4$ cross-linker ratio show high drug loading capacity for both the drugs compared with $1: 6$ crosslinker ratio. The $\gamma$-CD-NSPs-1:4 and $\beta$-CD-1:4 showed high loading efficiency for doxorubicin and captopril, respectively. Overall, the loading capacities of $\beta$-CD-NSPs and $\gamma$-CD-NSPs were the highest for both the drugs due to the better entrapment efficiency of monomer CDs, which also contributed in relative payloads. It is important to note that a negligible or minor difference was recorded in drug loading capacities of derivative NSPs on changing the cross-linker ratio from $1: 4$ to $1: 6$, unlike the parent NSPs which showed a significant alternation. This can be attributed to the limited hydroxyl groups for the cross-linking reaction and the change in the cross-linker ratio that failed to alter the structure, which explains the significant role of external cavities in drug loading. The images of lyophilized samples of doxorubicin-loaded CD-NSPs are presented in Fig. S5. $\dagger$ Overall, the crystalline samples showed low or negligible payload in some cases, which might be due to the proper long channels of specific sizes hindering the inflow of drugs, unlike with doxorubicin. It was reported that the $\alpha$-CD can only partially include captopril, while the $\beta$-CD is able to include an entire molecule inside the cavity, which can explain the large difference in payload efficiency of the respective combinations. ${ }^{29}$ These alternations might be due to the physicochemical properties of CDs and drugs also. The effect of cross-linker ratio, which resulted in the alternation of inner cavity sizes of NSPs can be easily estimated from loading data. Overall, the payload efficiency of each type of NSPs can be largely varied with a specific drug.

\section{Conclusions}

A facile synthesis of crystal or amorphous CD-NSPs of diverse physicochemical properties using DPC as a cross-linker was reported and compared in every aspect. The control of structural arrangements through derivatization of CDs was successfully implemented herein. The SEM images captured after lyophilization revealed the very different morphologies and highly porous structures of NSPs including the perfect spheres of M- $\beta$-CD-NSPs. The complete transformation of crystallinity from parent $\operatorname{CDs}(\alpha, \beta$ and $\gamma)$ and $\beta$-CD derivatives (M- $\beta$-CD, HP- $\beta-C D$ and SBE- $\beta$-CD) to their relative NSPs was recorded. The oven-dried NSPs completely lost the original structure after shrinking and aggregated into a very rigid structure. In addition, payload efficiency of all NSPs for doxorubicin and captopril was checked and compared. It was proven here that the crystallinity of NSPs can be controlled by CDs, which altered the drug loading efficiency and other physicochemical properties to a large extent. Altogether, the new class of NSPs proved highly diverse and showed a significant variation in structural properties and drug loading efficiencies. The presented results provided new possibilities for the selection and use of CD-NSPs as drug delivery systems. The 
reported CD-NSPs will be proven as a new alternatives or choices for the efficient delivery of hydrophobic or hydrophilic drugs.

\section{Acknowledgements}

The authors are grateful for the financial support from the National Natural Science Foundation of China (No. 81430087) and National Science and Technology Major Project (2013ZX09402103).

\section{Notes and references}

1 G. Crini, Chem. Rev., 2014, 114, 10940-10975.

2 G. Tiwari, R. Tiwari and A. K. Rai, J. Pharm. BioAllied Sci., 2010, 2, 72-79.

3 B. Gidwani and A. Vyas, BioMed Res. Int., 2015, 198268.

4 J. R. Lakkakula and R. W. Maçedo-Krause, Nanomedicine, 2014, 9, 877-894.

5 J. Zhang and P. X. Ma, Adv. Drug Delivery Rev., 2013, 65, 12151233.

6 M. K. Smith, S. R. Angle and B. H. Northrop, J. Chem. Educ., 2015, 92, 368-372.

7 B. Gidwani and A. Vyas, Colloids Surf., B, 2014, 114, 1301377.

8 F. Trotta, F. Caldera, C. Dianzani, M. Argenziano, G. Barrera and R. Cavalli, ChemPlusChem, 2016, 81, 439-443.

9 G. Tejashri, B. Amrita and J. Darshan, Acta Pharm., 2013, 63, 335-358.

10 R. Cavalli, F. Trotta and W. J. Tumiatti, J. Inclusion Phenom. Macrocyclic Chem., 2006, 56, 209-213.

11 S. J. Torne, K. A. Ansari, P. R. Vavia, F. Trotta and R. Cavalli, Drug Delivery, 2010, 17, 419-425.

12 S. Swaminathan, L. Pastero, L. Serpe, F. Trotta, P. Vavia, D. Aquilano, M. Trotta, G. Zara and R. Cavalli, Eur. J. Pharm. Biopharm., 2010, 74, 193-201.

13 K. A. Ansari, P. R. Vavia, F. Trotta and R. Cavalli, AAPS PharmSciTech, 2011, 12, 279-286.
14 S. Swaminathan, P. R. Vavia, F. Trotta and S. Torne, J. Inclusion Phenom. Macrocyclic Chem., 2007, 57, 89-94.

15 A. Singireddy and S. Subramanian, Part. Sci. Technol., 2016, 34, 341-346.

16 S. Sadjadi, M. M. Heravi and M. Daraie, Res. Chem. Intermed., 2017, 43, 843-857.

17 H. Li, B. Meng, S. H. Chai, H. Liu and S. Dai, Chem. Sci., 2016, 7, 905-909.

18 P. L. Meo, G. Lazzara, L. Liotta, S. Riela and R. Noto, Polym. Chem., 2014, 5, 4499-4510.

19 A. Alsbaiee, B. J. Smith, L. Xiao, Y. Ling, D. E. Helbling and W. R. Dichtel, nature, 2016, 529, 190-194.

20 M. Russo, M. L. Saladino, D. C. Martino, P. L. Meo and R. Noto, RSC Adv., 2016, 6, 49941-49953.

21 R. Z. Ahmed, P. Gunjan and Z. Zaheer, Drug Dev. Ind. Pharm., 2013, 39, 1263-1272.

22 F. Castiglione, V. Crupi, D. Majolino, A. Mele, W. Panzeri, B. Rossi, F. Trotta and V. Venuti, J. Inclusion Phenom. Macrocyclic Chem., 2013, 75, 247-254.

23 V. Crupi, D. Majolino, A. Mele, L. Melone, C. Punta, B. Rossi and F. Toraldo, Soft Matter, 2014, 10, 2320-2326.

24 F. Castiglione, V. Crupi, D. Majolino, A. Mele, B. Rossi, F. Trotta and V. Venuti, J. Raman Spectrosc., 2013, 44, 1463-1469.

25 P. Shende, Y. A. Kulkarni, R. S. Gaud, K. Deshmukh, R. Cavalli, F. Trotta and F. Caldera, J. Pharm. Sci., 2015, 104, 1856-186326.

26 V. Singh, T. Guo, L. Wu, J. Xu, B. Liu, R. Gref and J. Zhang, RSC Adv., 2017, 7, 20789-20794.

27 O. Tacar, P. Sriamornsak and C. R. Dass, J. Pharm. Pharmacol., 2013, 65, 157-170.

28 Y. Yusof, D. T. Tan, O. K. Arjomandi, G. Schenk and R. P. McGeary, Bioorg. Med. Chem. Lett., 2016, 26, 15891593.

29 Y. Ikeda, S. Motoune, T. Matsuoka, H. Arima, F. Hirayama and K. Uekama, J. Pharm. Sci., 2002, 91, 2390-2398. 